\title{
Reversing Chemotherapy Resistance by a Synergy Between Lysosomal pH-Activated Mitochondrial Drug Delivery and Erlotinib-Mediated Drug Efflux Inhibition
}

Furong Cheng,b,c, Qingqing Pan, ${ }^{a}$ Wenxia Gao, ${ }^{\mathrm{d}}$ Yuji Pu, ${ }^{\mathrm{a}}$ Kui Luo, ${ }^{\mathrm{e}}$ Bin $\mathrm{He}^{\mathrm{a}}$

${ }^{a}$ National Engineering Research Center for Biomaterials, College of Biomedical Engineering, Sichuan University, Chengdu 610064, China.

${ }^{\mathrm{b}}$ Center for Translational Medicine, The Second Affiliated Hospital, Guangzhou Medical University, Guangzhou 510260, China

c Department of Pharmaceutics, College of Pharmacy, Virginia Commonwealth University, Richmond 23219, Virginia, United States

${ }^{d}$ College of Chemistry \& Materials Engineering, Wenzhou University, Wenzhou 325027, China

${ }^{\text {e }}$ Huaxi MR Research Center, Department of Radiology, West China Hospital, Functional and molecular imaging Key Laboratory of Sichuan Province, Sichuan University, Chengdu, 610041, China

E-mail: yjpu@scu.edu.cn 
Characterizations. The ${ }^{1} \mathrm{H}$ nuclear magnetic resonance $\left({ }^{1} \mathrm{H}\right.$ NMR) and fourier transform infrared (FTIR) spectra were recorded on an NMR spectrometer (Bruker Avance-400 MHz) and an FTIR spectrometer (Thermo Fisher Scientific Nicolet 8700), respectively. The transmission electron microscope (TEM, JEOL JEM 2100F) was used to observe the size and morphology of polymeric nanoparticles. The sizes and zeta potentials of the nanoparticles were determined by a dynamic light scattering (DLS) spectrometer (Malvern Zetasizer Nano ZS) at $25^{\circ} \mathrm{C}$.

Preparation of Blank and Drug-Loaded Nanoparticles. For the blank nanoparticles, PKCF in water (20 mg mL $\left.\mathrm{mL}^{-1}, 0.5 \mathrm{~mL}\right)$ was added to distilled water $(9.5 \mathrm{~mL})$. After stirring overnight, the blank nanoparticles (NPs) were obtained after freeze-drying.

Drug-loaded nanoparticles were prepared by a dialysis method. We prepared a series of nanoparticles (DE-NPs-0.15, DE-NPs-0.3, DE-NPs-0.45) with different DOX/Erlo feeding weight ratios (Table S1). PKCF (10 mg), DOX (1.25 mg), and Erlo (0.5 mg, $0.88 \mathrm{mg}$, and $1.25 \mathrm{mg}$ for DENPs-0.15, DE-NPs-0.3, and DE-NPs-0.45, respectively) were dissolved in a mixed solvent of DMSO and $\mathrm{H}_{2} \mathrm{O}\left(V / V\right.$, DMSO$\left./ \mathrm{H}_{2} \mathrm{O}=8 / 3,0.55 \mathrm{~mL}\right)$. Then the mixture was added to $9.85 \mathrm{~mL}$ distilled water. After stirring overnight, the solution was dialyzed (MWCO 1000) against distilled water for 12 h. After centrifugation, the solution was lyophilized to obtain DOX (D) and Erlo (E) co-loaded nanoparticles (DE-NPs). The whole procedure was performed in the dark. DOX-loaded nanoparticles (D-NPs) were prepared similarly. The amount of encapsulated DOX was measured by UV-vis spectroscopy (Perkin-Elmer Ltd Co., USA) at $483 \mathrm{~nm}$. The amount of encapsulated Erlo was determined by high performance liquid chromatography (HPLC, Agilent HPLC System, USA). The optimized mobile phase was a mixed solvent of $\mathrm{H}_{2} \mathrm{O}$ (with $0.1 \%$ TFA) and acetonitrile ( $V / V$,

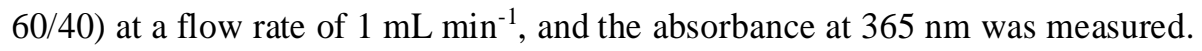


pH-Responsiveness of NPs. (1) Acid-base titration. Briefly, NPs solution (2 mg mL $\mathrm{m}^{-1}, 3 \mathrm{~mL}$ ) was adjusted initially to $\mathrm{pH} 10$ by $0.01 \mathrm{M} \mathrm{NaOH}$. The suspension was then titrated to $\mathrm{pH} 3.0$ with aliquots of $10 \mu \mathrm{L}$ of $0.01 \mathrm{M} \mathrm{HCl}$. The $\mathrm{pH}$ value of the suspension was monitored by a $\mathrm{pH}$-meter (InLab ${ }^{\circledR}$ Expert Pro, Mettler Toledo). (2) Fluorescence spectrophotometry with OPA derivatization. NPs solution (2 $\mathrm{mg} \mathrm{mL}^{-1}, 3 \mathrm{~mL}$ ) was mixed with the same volume of $0.02 \mathrm{M}$ different buffers (PBS: $\mathrm{pH}=7.4$, ABS (acetate buffer solution): $\mathrm{pH}=6.5$ or 5.0 ). Then the mixtures with different $\mathrm{pH}$ values were placed in a water bath shaker $\left(120 \mathrm{rpm}, 37^{\circ} \mathrm{C}\right)$. At different time points, $1 \mathrm{~mL}$ micelle solution was taken out, then $100 \mu \mathrm{L}$ of $0.4 \mathrm{M}$ borate saline buffer ( $\mathrm{pH}=9.5$ ) and $10 \mu \mathrm{L}$ of OPA buffer ( $5 \mathrm{mg}$ OPA dissolved in $0.1 \mathrm{~mL}$ ethanol, and $5 \mu \mathrm{L}$ 3-mercaptopropionic acid were mixed with $0.9 \mathrm{~mL} 0.4$ M borate saline buffer.) was added. After a 3-min-reaction, the fluorescence spectroscopy of mixture was measured. (3) Zeta potential changes. NPs solution (2 mg mL $\left.{ }^{-1}, 1.5 \mathrm{~mL}\right)$ was mixed with the same volume of $0.02 \mathrm{M}$ different buffers (PBS: $\mathrm{pH}$ 7.4, ABS: $\mathrm{pH} 6.5$ or 5.0). Then the mixtures were placed in a water bath shaker $\left(120 \mathrm{rpm}, 37^{\circ} \mathrm{C}\right)$ and $1 \mathrm{~mL}$ of the mixture was taken out for DLS test at the set time points.

Cell Culture. L929, MCF-7, MCF-7/ADR cells were cultured with DMEM solution (10\% FBS, 1\% penicillin-streptomycin) at a incubator containing $5 \% \mathrm{CO}_{2}$ at $37^{\circ} \mathrm{C}$. For MCF-7/ADR cells, DMEM solution also containing $1 \mu \mathrm{g} \mathrm{mL^{-1 }}$ DOX to maintain the drug resistance.

In Vitro Cellular Uptake. CLSM and flow cytometry were used to study the In vitro cellular uptake behavior. For the CLSM study, MCF-7/ADR (or MCF-7) cells were seeded on glass dishes ( $d=35 \mathrm{~mm}$ ) and treated with different drug formulations for 2 and $6 \mathrm{~h}$ (DOX: $10 \mu \mathrm{g} \mathrm{mL} \mathrm{L}^{-1}$ ), cells were washed with cold PBS twice and stained with Hoechst 33342. Then the cells were washed with cold PBS twice for CLSM observation. For the flow cytometry, MCF-7/ADR cells were treated with different drug 
formulations for 2 and $6 \mathrm{~h}$ (DOX: $10 \mu \mathrm{g} \mathrm{mL} \mathrm{L}^{-1}$ ), harvested and washed with PBS twice for flow cytometry.

Lysosomal Escape. MCF-7/ADR cells were cultured on glass dishes $(d=35 \mathrm{~mm})$. After incubation with DE-NPs-0.45 for $2 \mathrm{~h}$ (DOX: $10 \mu \mathrm{gL}^{-1}$ ). After replacing with fresh medium and further incubation for 2, 6, and $24 \mathrm{~h}$, cells were washed twice with PBS twice, stained with LysoSensor Yellow/Blue DND160 (Life Technologies Corporation, USA) for 30 min, Then the cells were washed with PBS twice and observed by CLSM.

Drug Efflux Assay. Cells were incubated with DOX, D-NPs, or DE-NPs-0.45 for 4 h (DOX: $10 \mu \mathrm{g}$ $\mathrm{mL}^{-1}$ ), followed by incubation with drug-free medium for $20 \mathrm{~h}$. The cells were washed with cold PBS for CLSM study. For cytometry study, MCF-7/ADR cells were treated with DOX, D-NPs or DE-NPs-0.45 containing medium for $2 \mathrm{~h}$ (DOX: $10 \mu \mathrm{g} \mathrm{mL}^{-1}$ ), followed by incubation with drug-free medium for 0 and $22 \mathrm{~h}$. Cells were collected for flow cytometry. The mean fluorescence intensities were calculated from three parallel experiments.

Western Blot Analysis. MCF-7/ADR cells were separately inoculated into 6-well plates. After a $12 \mathrm{~h}$ incubation, cells were treated with DOX, DOX+Erlo (D+E), and DE-NPs-0.45 for $24 \mathrm{~h}$ and harvested for western blot test (DOX: $10 \mu \mathrm{g} \mathrm{mL}^{-1}$, Erlo: $4.5 \mu \mathrm{g} \mathrm{mL} \mathrm{m}^{-1}$ ).

Molecular Docking. The structures of P-gp were downloaded from the RCSB protein data bank (PDB: $4 \mathrm{M} 1 \mathrm{M}, 6 \mathrm{C} 0 \mathrm{~V})$. The structures were purified by removing all the heteroatoms and solvent molecules. Erlo was subjected to energy minimization using Chem3D. Erlo was docked within the NBDs of P-gp using Autodock 4.0 and the Lamarkian genetic algorithm. Optimal docking was selected based on considerations of binding free energy. Docking poses generated by Autodock4 were directly loaded into PyMOL for further analysis and 3D export.

Cell Cycle and Apoptosis Analysis. MCF-7/ADR cells were cultured in $2 \mathrm{~mL}$ culture medium 
containing Erlo, DOX, D-NPs, or DE-NPs-0.45 for 24 h (DOX: $10 \mu \mathrm{gL}^{-1}$, Erlo: $4.5 \mu \mathrm{g} \mathrm{mL} \mathrm{m}^{-1}$ ). Then PI/RNase staining buffer and Annexin V-FITC/PI kit were used for cell cycle and apoptosis analysis studies as our previous study. ${ }^{1}$

In Vitro Penetration in 3D Cell Spheroids. 48-well plate was pretreated with $150 \mu \mathrm{L}$ of sterile $2 \%$ agarose (dissolved in PBS), then MCF-7/ADR cells were seeded and cultured a few days to form 3D tumor cell spheroids preparation. Then the 3D tumor cell spheroids were transferred to glass dishes $(d=$ $35 \mathrm{~mm}$ ) and treated with DOX or DE-NPs-0.45 for $6 \mathrm{~h}\left(\mathrm{DOX}: 10 \mu \mathrm{g} \mathrm{mL}^{-1}\right.$, Erlo: $4.5 \mu \mathrm{g} \mathrm{mL} \mathrm{L}^{-1}$ ), following washed with PBS twice and observed by CLSM.

\section{References}

(1) Cheng, F.; Peng, X.; Meng, G.; Pu, Y.; Luo, K.; He, B., Poly (ester-thioether) Microspheres CoLoaded with Erlotinib and $\alpha$-Tocopheryl Succinate for combinational Therapy of Non-Small Cell Lung Cancer. J. Mater. Chem. B 2020, 8, 1728-1738. 
Table S1. DOX and Erlo loading contents in NPs.

\begin{tabular}{cccc}
\hline NPs & $\begin{array}{c}\text { Feeding weight } \\
\text { ratios of DOX/Erlo }\end{array}$ & DLCs ${ }^{\mathrm{a}}$ of DOX & DLCs of Erlo \\
\hline D-NPs & $1: 0$ & $6.6 \%$ & - \\
DE-NPs-0.15 & $1: 0.4$ & $4.7 \%$ & $0.7 \%$ \\
DE-NPs-0.3 & $1: 0.7$ & $5.1 \%$ & $1.5 \%$ \\
DE-NPs-0.45 & $1: 1$ & $5.8 \%$ & $2.6 \%$ \\
\hline
\end{tabular}

${ }^{a}$ DLCs: drug loading contents.

Table S2. CI values of different drug formulations.

\begin{tabular}{ccccccc}
\hline \multirow{2}{*}{$\mathrm{CI}_{\mathrm{n}}$} & \multicolumn{2}{c}{ MCF-7 } & & \multicolumn{2}{c}{ MCF-7/ADR } \\
\cline { 2 - 3 } \cline { 5 - 6 } & $\mathrm{CI}_{25}$ & $\mathrm{CI}_{50}{ }^{\mathrm{a}}$ & & $\mathrm{CI}_{25}$ & $\mathrm{CI}_{50}{ }^{\mathrm{a}}$ \\
\hline DE-NPs-0.15 & 1.25 & $<0.72$ & & 0.15 & $<0.25$ \\
DE-NPs-0.3 & 1.05 & $<0.94$ & & 0.13 & $<0.11$ \\
DE-NPs-0.45 & 0.63 & $<0.57$ & & 0.12 & $<0.07$ \\
\hline
\end{tabular}

${ }^{\mathrm{a}}$ Based on considering $100 \mu \mathrm{g} \mathrm{mL}^{-1}$ as the concentration for single Erlo to achieve $50 \%$ inhibition of cell proliferation.

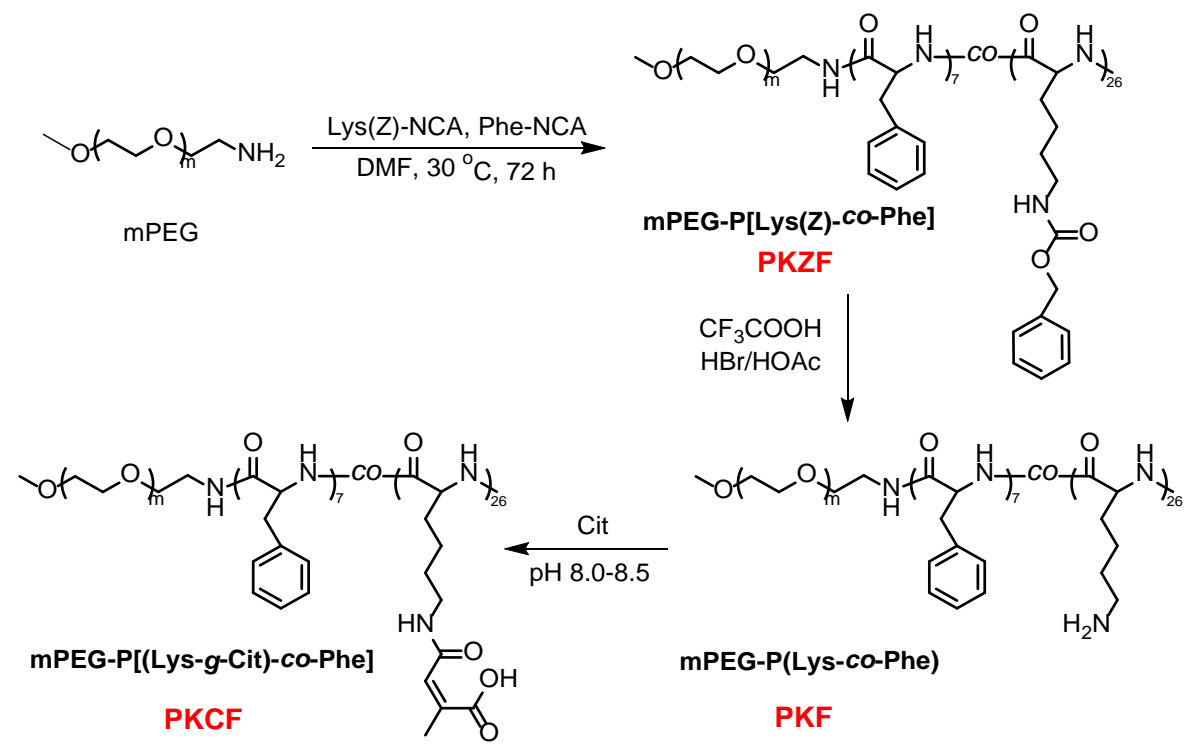

Scheme S1. Synthetic route of PKCF. 

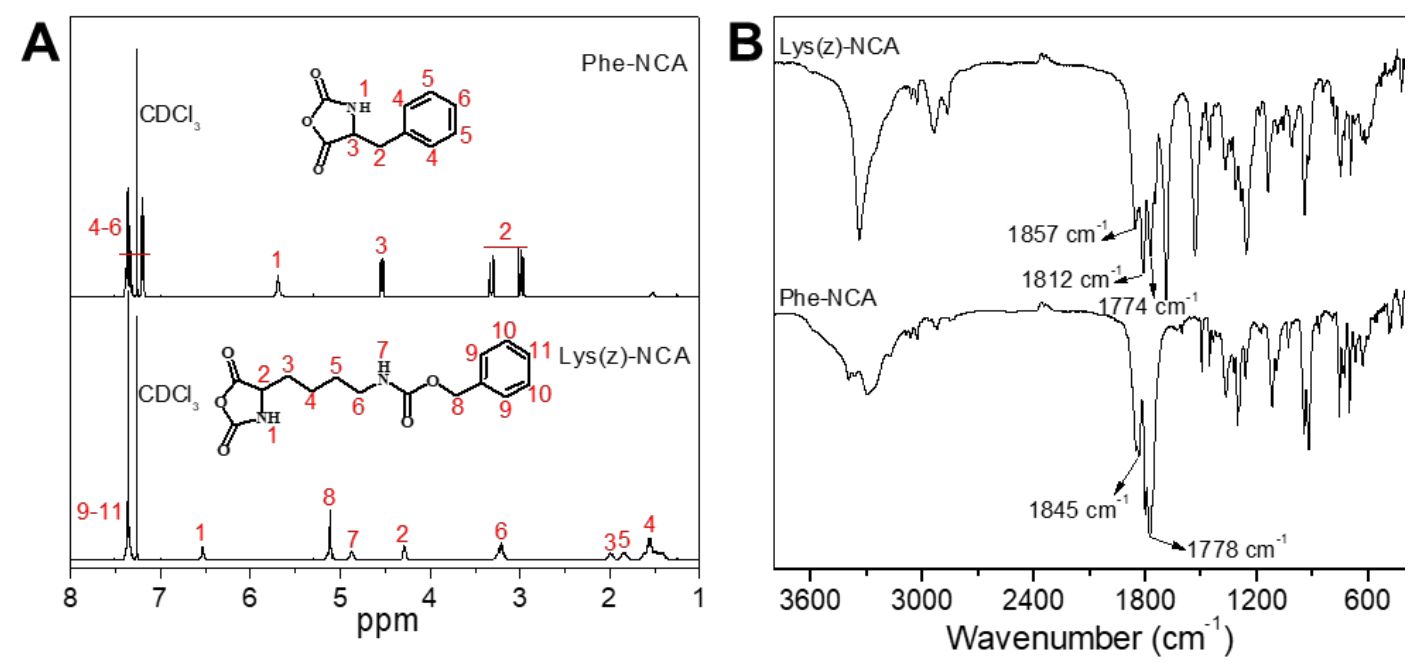

Figure S1. ${ }^{1} \mathrm{H}$ NMR (A) and FTIR (B) spectra of Phe-NCA and Lys(Z)-NCA.
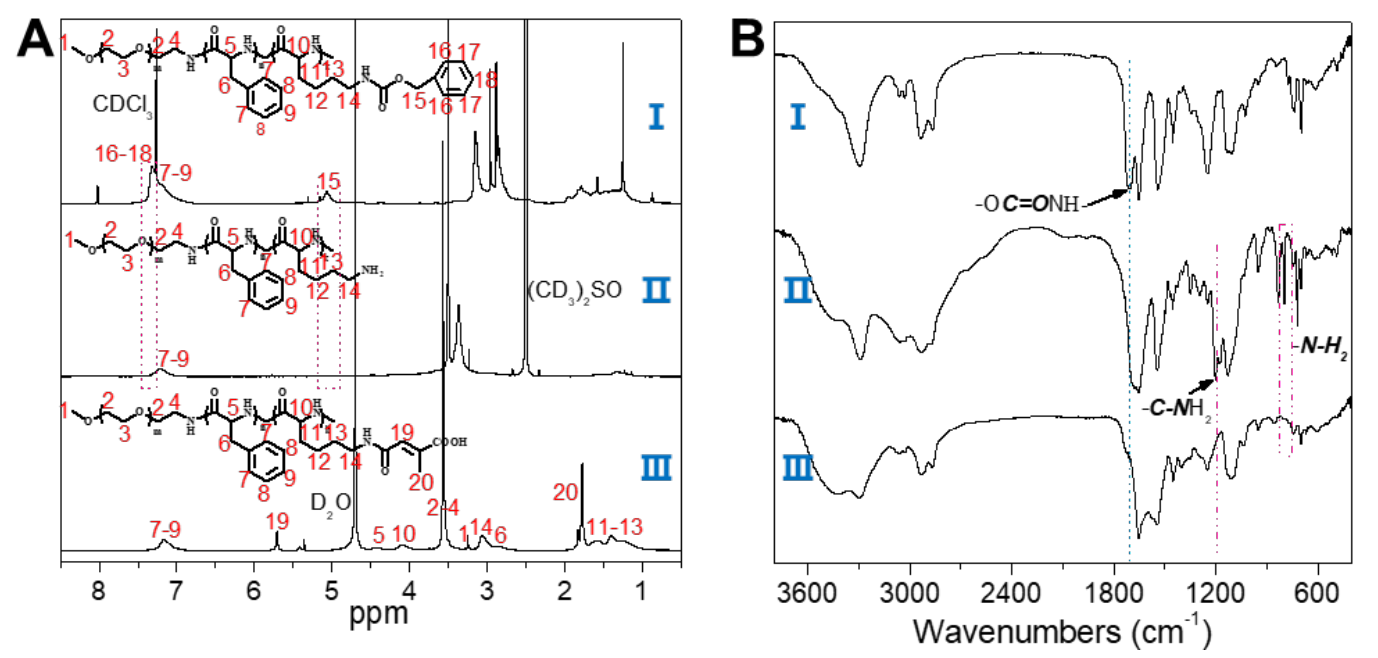

Figure S2. ${ }^{1} \mathrm{H}$ NMR (A) and FTIR (B) spectra of PKZF (I), PKF (II), and PKCF (III). 

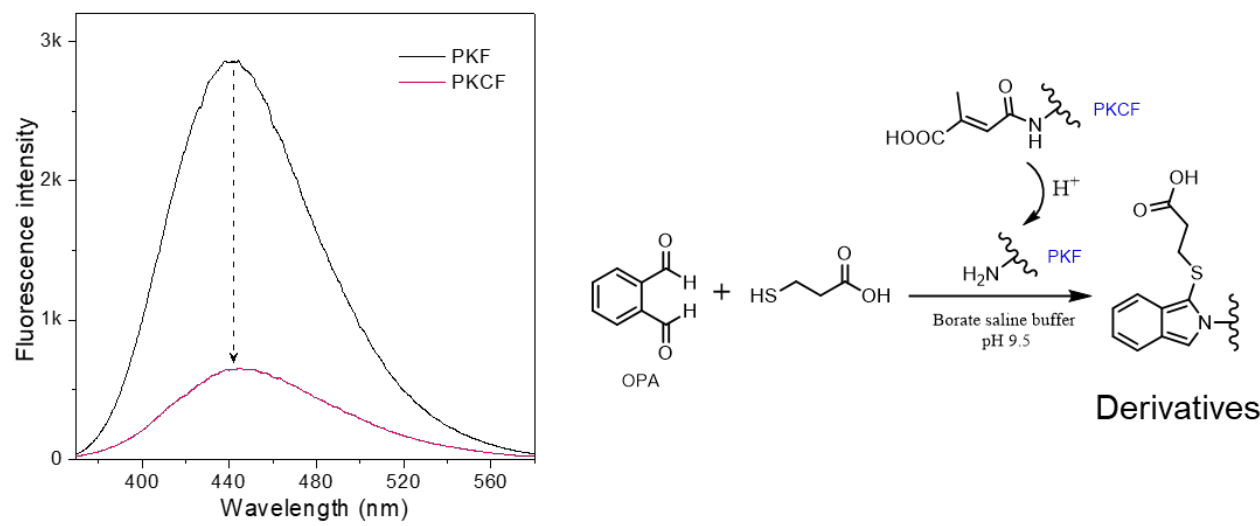

Figure S3. Fluorescence intensity of PKF and PKCF after the reaction with OPA buffer.

Right panel shows the chemical reaction to give fluorescent product.
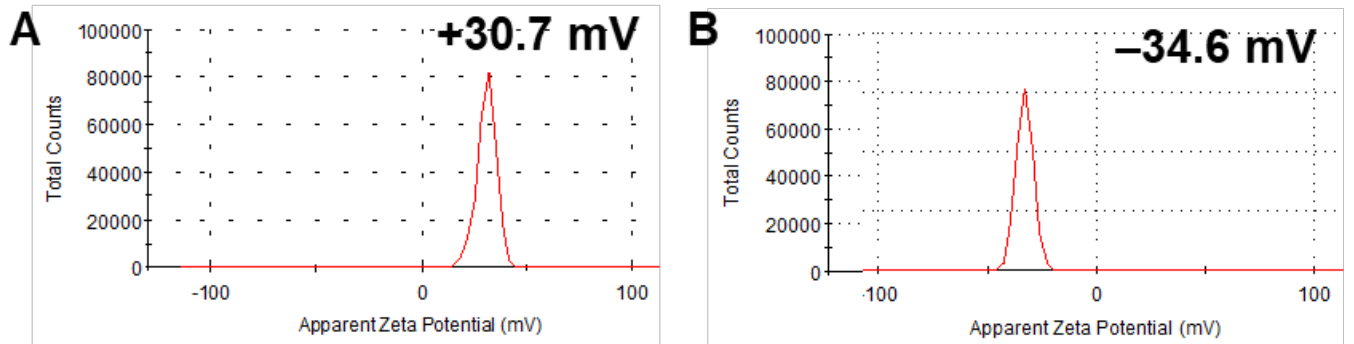

Figure S4. Zeta potentials of blank nanoparticles of PKF (A) and PKCF (B).

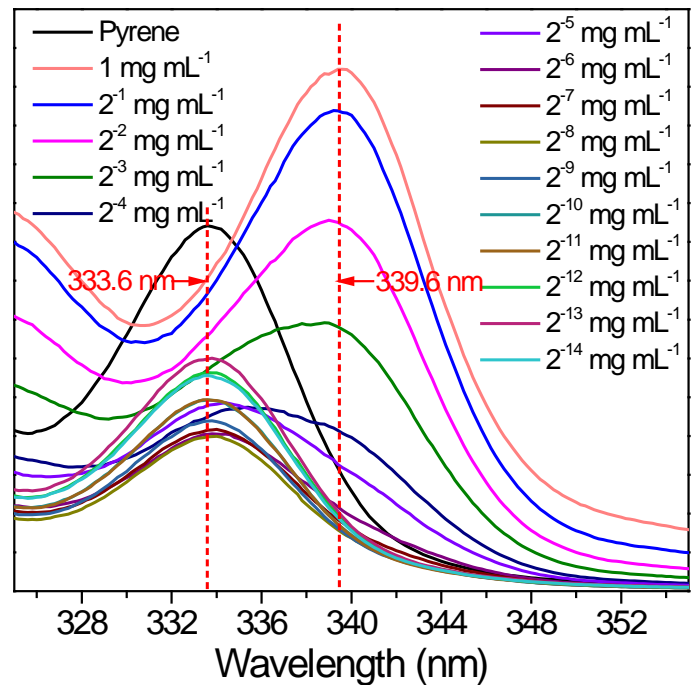

Figure S5. The fluorescence spectra of PKCF with different concentrations in saturated pyrene solution. 


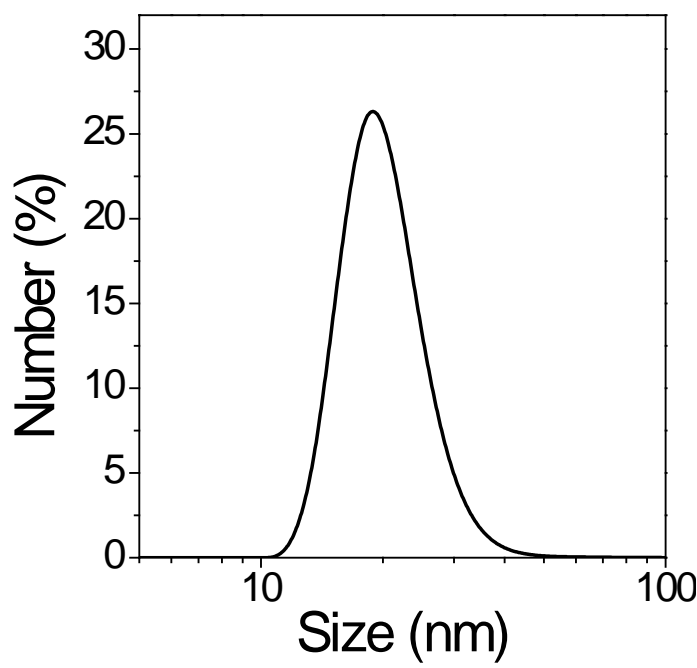

Figure S6. DLS size of blank PKCF NPs.

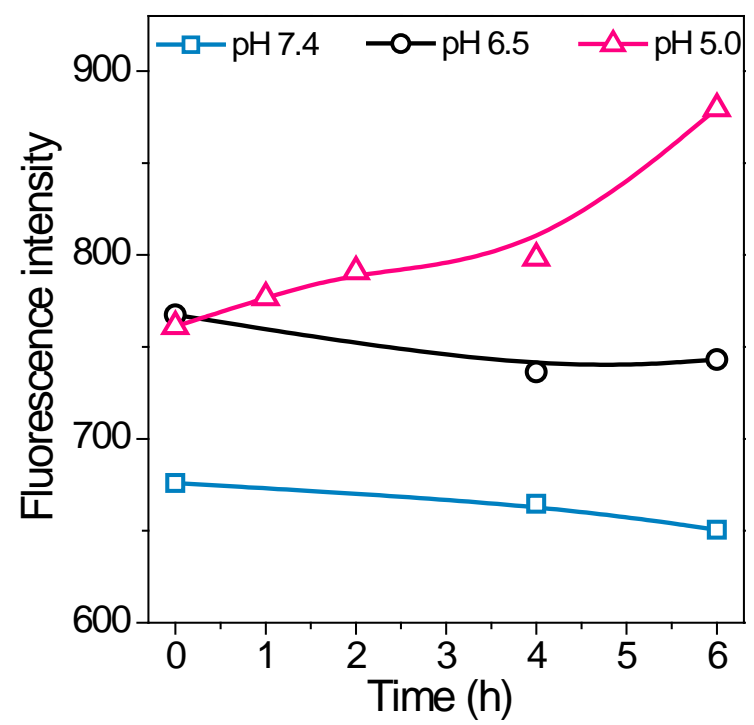

Figure S7. The changes of fluorescence intensity of NPs after the reaction with OPA buffer. 

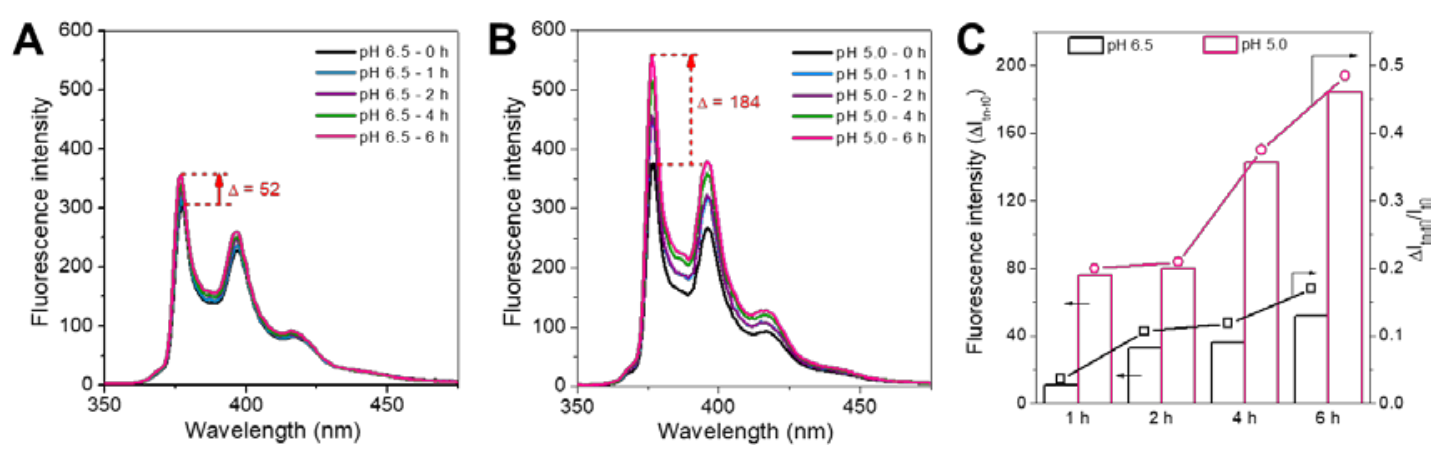

Figure S8. The fluorescence spectra of 1-aminomethylpyrene-loaded PKCF NPs after incubation in ABS 6.5 (A) and ABS 5.0 (B) over 6 h. (C) The changes of the fluorescence intensity $\left(\Delta I_{\mathrm{tn}-\mathrm{t} 0}=I_{\mathrm{tn}}-I_{\mathrm{t} 0}\right.$, where $I_{\mathrm{tn}}$ and $I_{\mathrm{t} 0}$ are the FL intensity of solution at $\mathrm{n}$ and $0 \mathrm{~h}$ ). The concentration of nanoparticles was $2 \mathrm{mg} \mathrm{mL}^{-1}$ with $\mathrm{Py}-\mathrm{NH} \mathrm{H}_{2} \cdot \mathrm{HCl}$ concentration was $10^{-5} \mathrm{M}$ to prepare $\mathrm{Py}-\mathrm{NH}_{2} \cdot \mathrm{HCl}$ loaded nanoparticles (Py-NPs). Then ABS (acetate buffer solution, $\mathrm{pH}=6.5$ and 5.0; ionic strength $=0.02 \mathrm{M}$ ) solutions were prepared with Py- $\mathrm{NH}_{2} \cdot \mathrm{HCl}$ concentration was $10^{-5} \mathrm{M}$. Then Py-NPs solution (2 mg mL1, $1.5 \mathrm{~mL}$ ) was mixed with the same volume of ABS ( $\mathrm{pH} 6.5$ and 5.0, $0.02 \mathrm{M}$ ). Then the mixtures were placed in a shaking bed $\left(37^{\circ} \mathrm{C}, 120 \mathrm{rpm}\right)$ and test by fluorescence spectra (F-7000, Hitachi Co. Japan) at the set time points. 

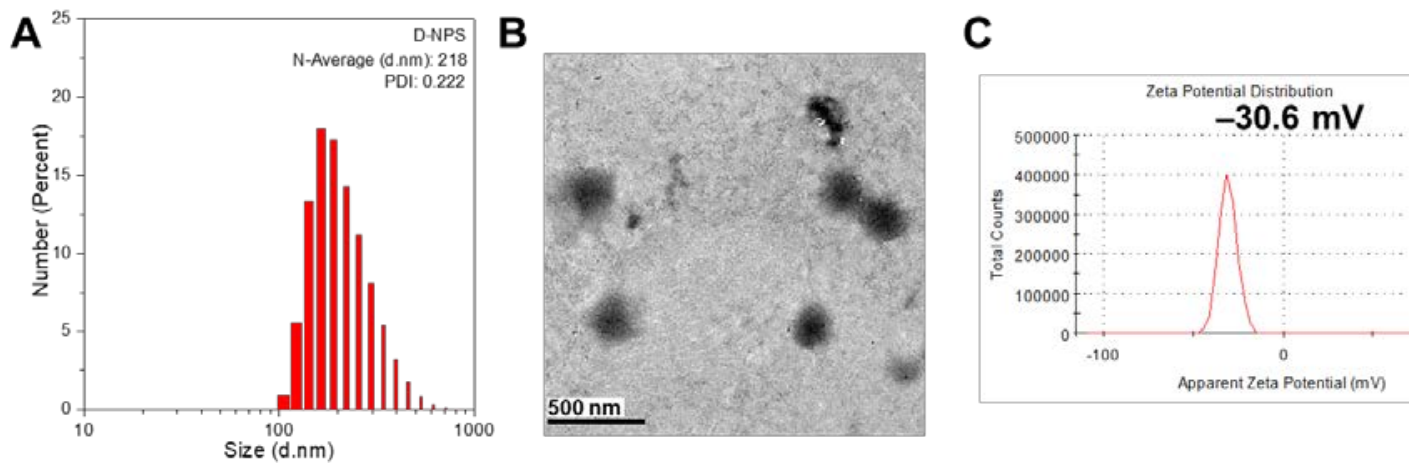

Figure S9. The (A) size distribution, (B) TEM image, and (C) zeta potential of D-NPs.

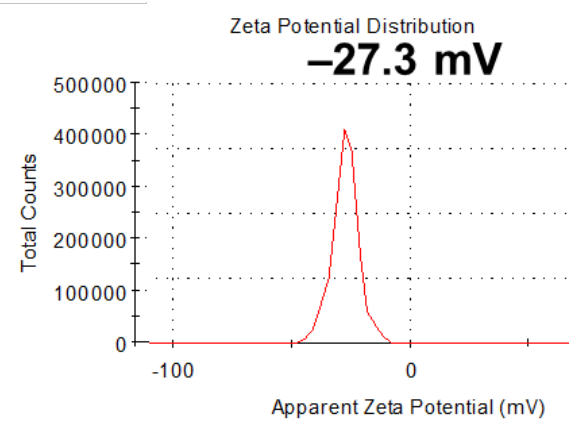

Figure S10. Zeta potential of DE-NPs-0.45.
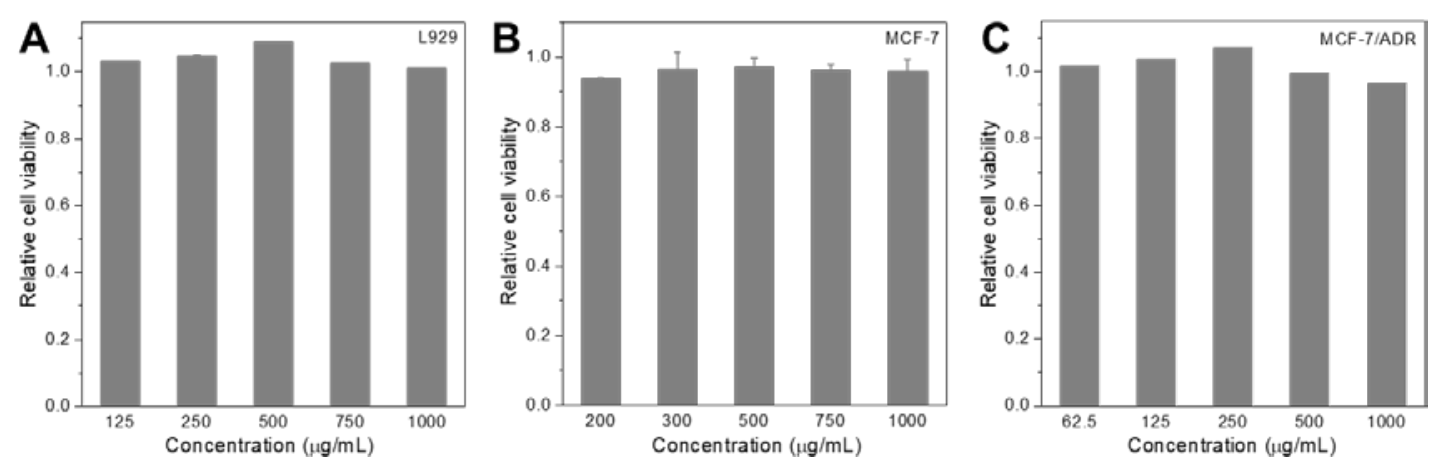

Figure S11. Viability of L929 (A), MCF-7 (B), and MCF-7/ADR (C) cells after treatment with blank polymeric NPs for $48 \mathrm{~h}, n=5$. 

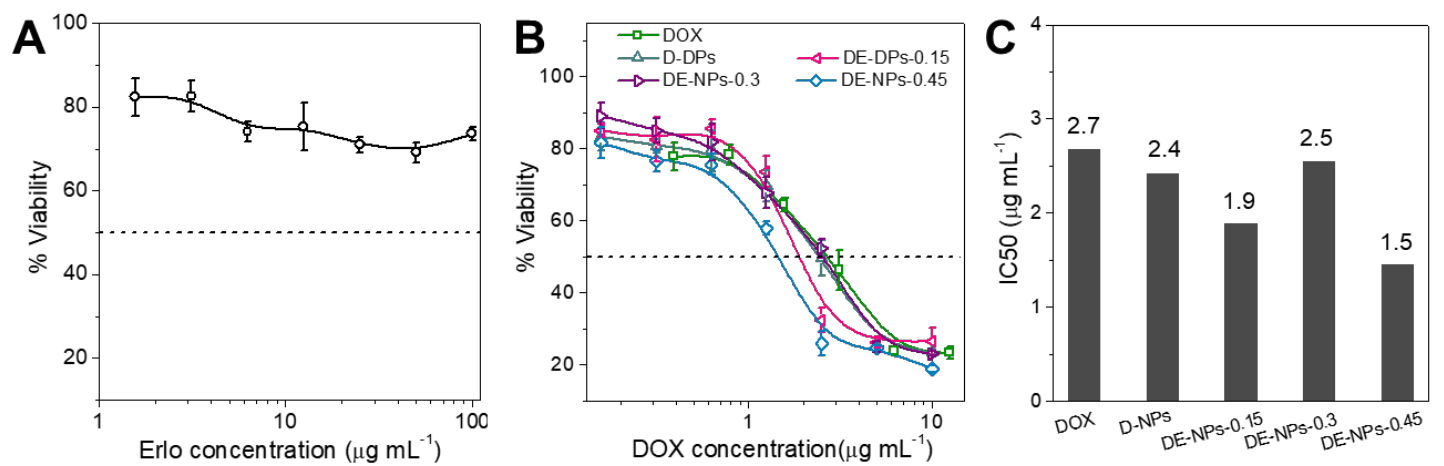

Figure S12. The cytotoxicity of erlotinib (A) and NPs (B) against MCF-7 cells after co-incubation for $48 \mathrm{~h}, n=5$. (C) IC50 values of various formulations to MCF-7 cells.
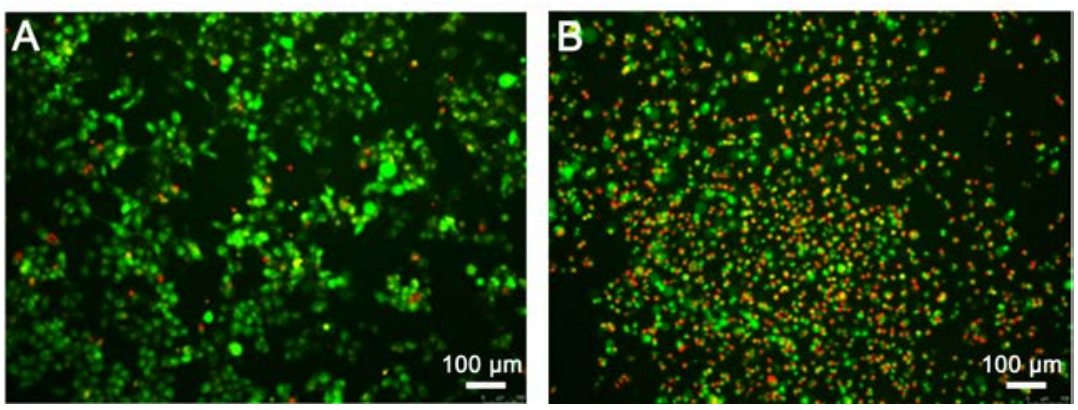

Figure S13. Live/dead staining images of MCF-7/ADR cells treated with D-NPs (A) and DE-NPs-0.15 (B) for $48 \mathrm{~h}$; the concentration of DOX was $5 \mu \mathrm{g} \mathrm{mL} \mathrm{m}^{-1}$. Live and dead cells were stained to be green and red, respectively. 

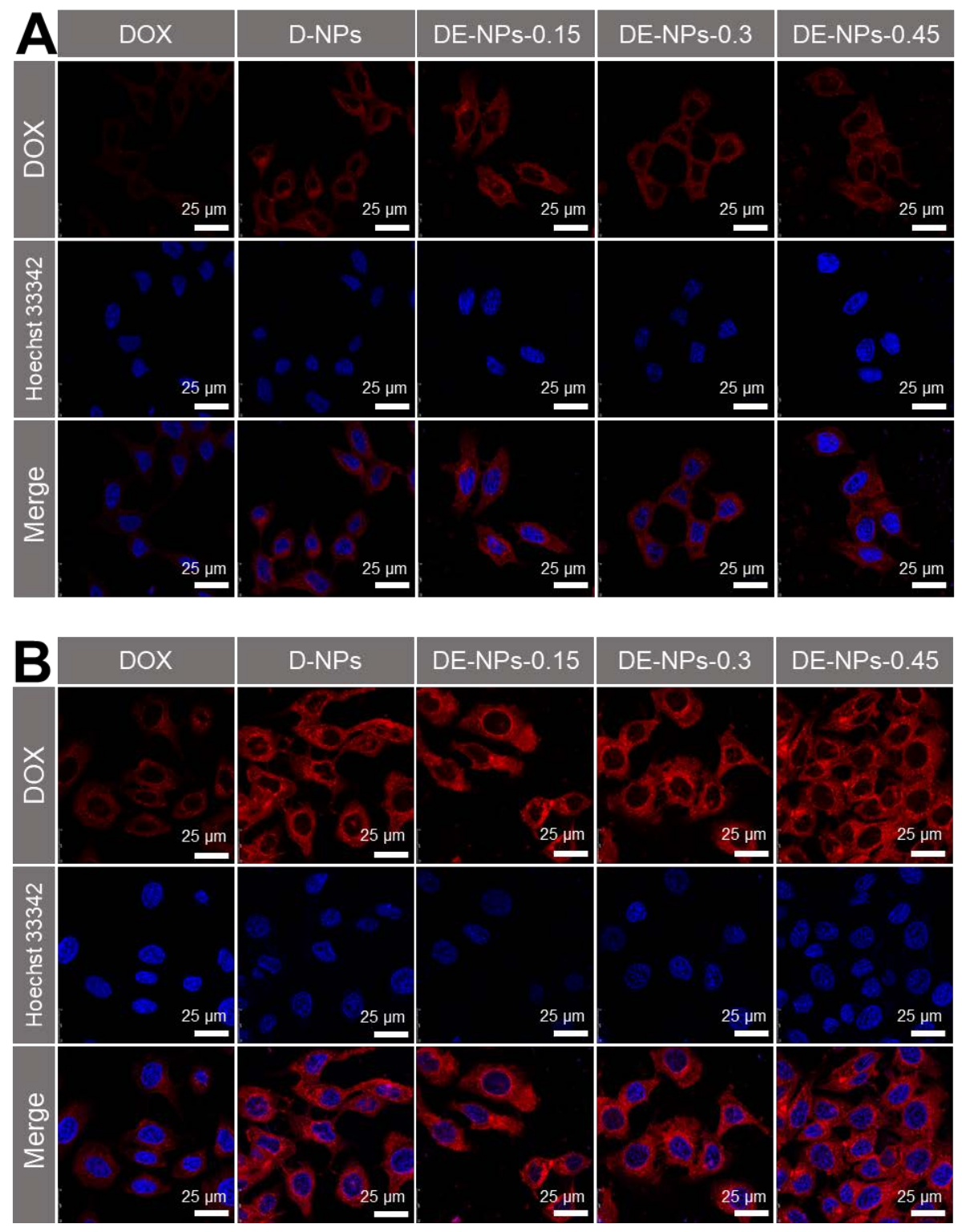

Figure S14. CLSM images of MCF-7 cells after co-incubation with different NPs for 2 (A) and $6 \mathrm{~h}(\mathrm{~B})$. The DOX concentration was $10 \mu \mathrm{g} \mathrm{mL}-1$. 


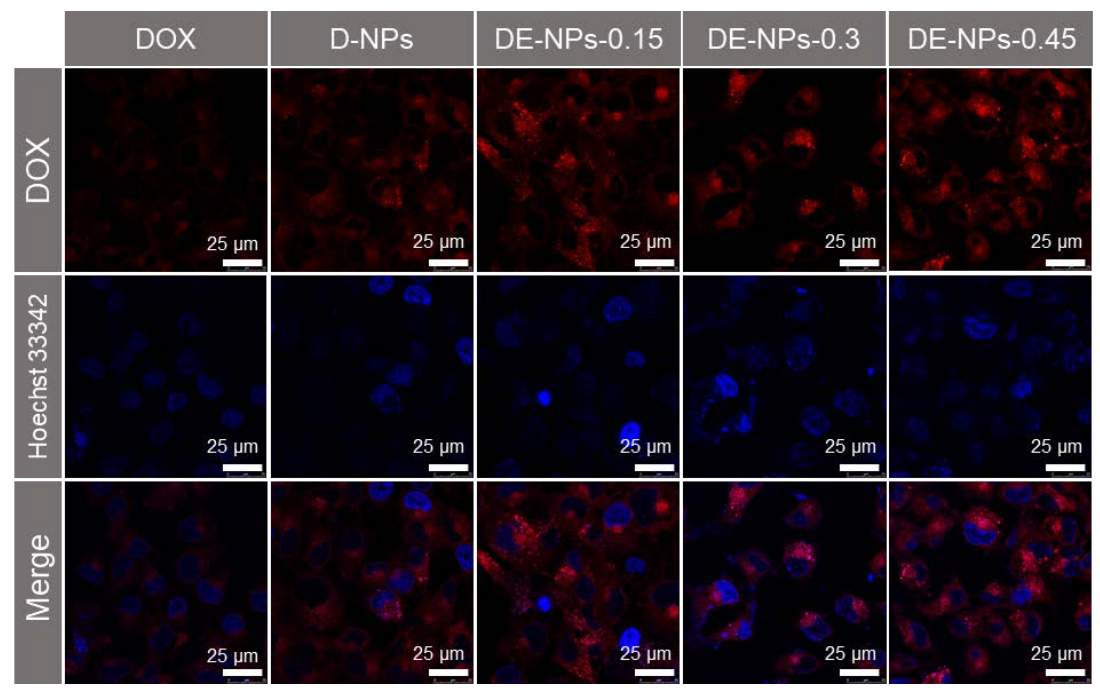

Figure S15. CLSM images of MCF-7/ADR cells after co-incubation with various NPs for $2 \mathrm{~h}$. The DOX concentration was $10 \mu \mathrm{g} \mathrm{mL}^{-1}$.
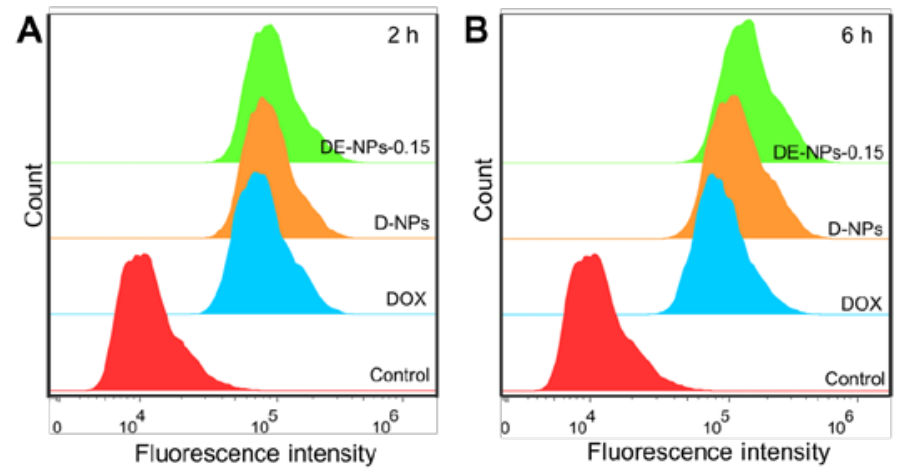

Figure S16. Flow cytometry results of MCF-7/ADR cells after co-incubation with free DOX and drug-loaded nanoparticles for 2 (A) and $6 \mathrm{~h}(\mathrm{~B})$. The DOX concentration was $10 \mu \mathrm{g} \mathrm{mL} \mathrm{m}^{-1}, n=3$. 


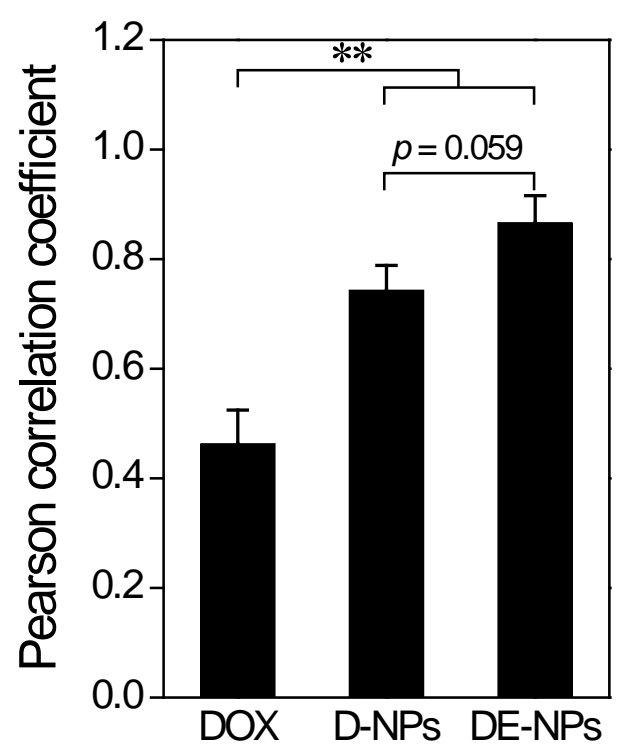

Figure S17. Pearson correlation coefficient between red and green fluorescence in MCF-7/ADR cells (Figure 3B) treated with different formulations. ${ }^{* *} p<0.01, n=3$.

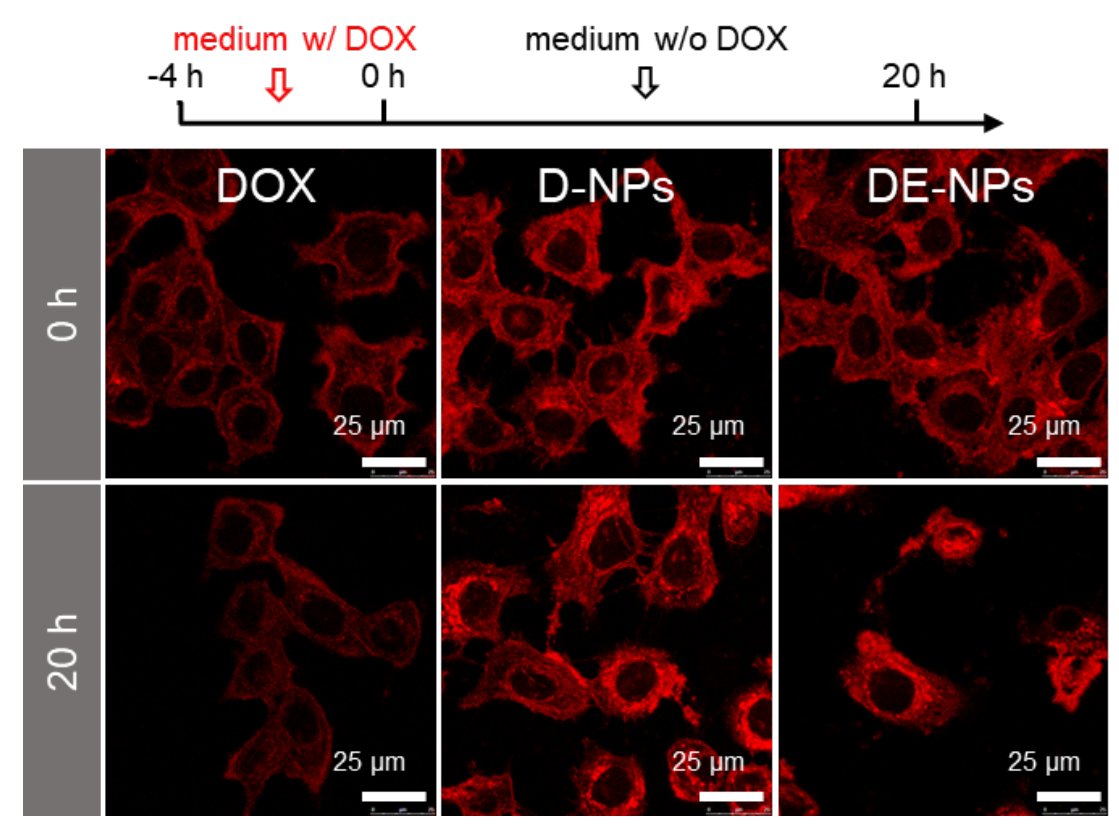

Figure S18. CLSM images of MCF-7 cells after co-incubation with fresh medium for 0 and $20 \mathrm{~h}$. The cells were pretreated with free DOX and drug-loaded NPs for 4 h. The DOX concentration was $10 \mu \mathrm{g} \mathrm{mL}-1$. 


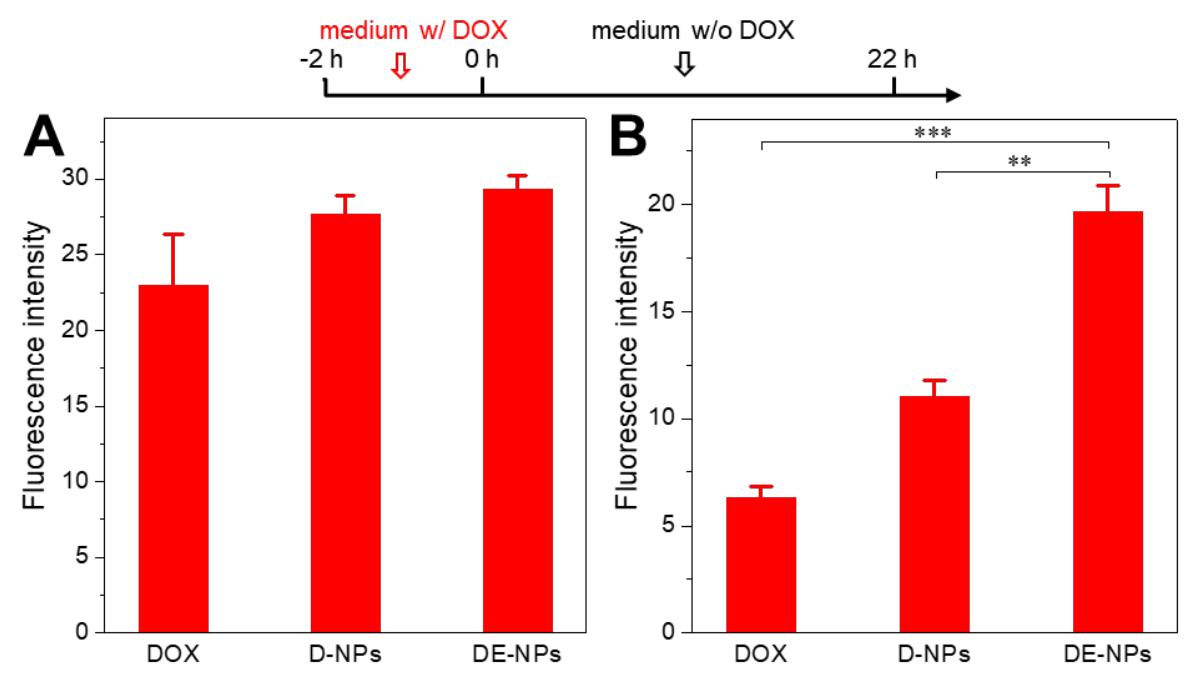

Figure S19. Flow cytometry results of MCF-7/ADR after co-incubation with fresh medium for $0 \mathrm{~h}(\mathrm{~A})$ and $22 \mathrm{~h}(\mathrm{~B}) .{ }^{* *} p<0.01, * * * p<0.001, n=3$.

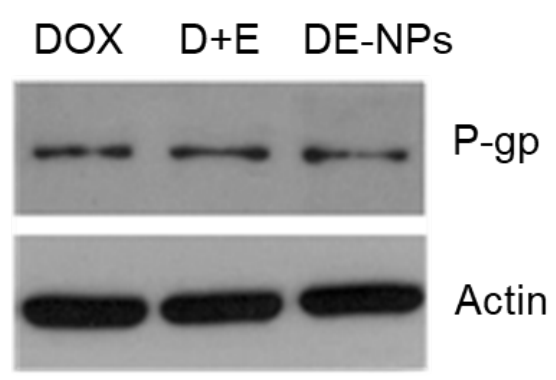

Figure S20. Western blot analysis for protein levels of P-gp expression in MCF-7/ADR cells after incubation with DOX, D+E, and DE-NPs for $24 \mathrm{~h}$.

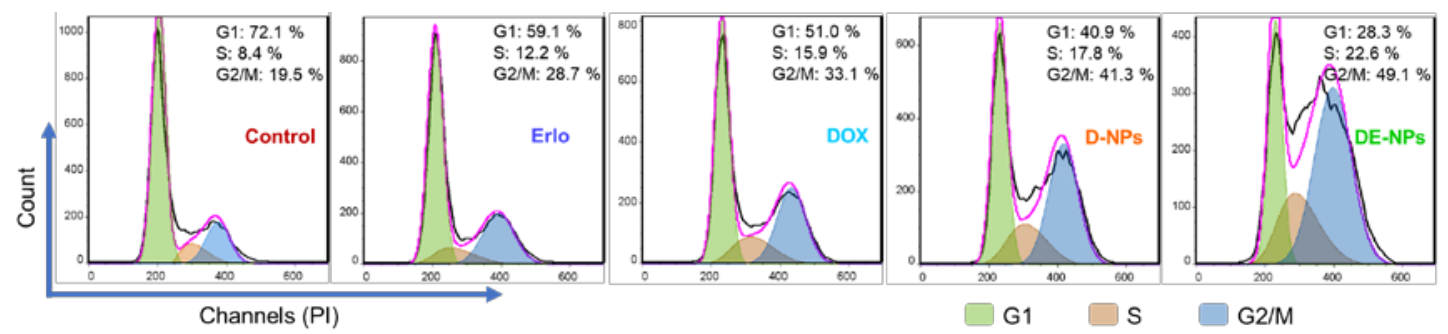

Figure S21. Cell cycle analysis of MCF-7/ADR after different treatments. 


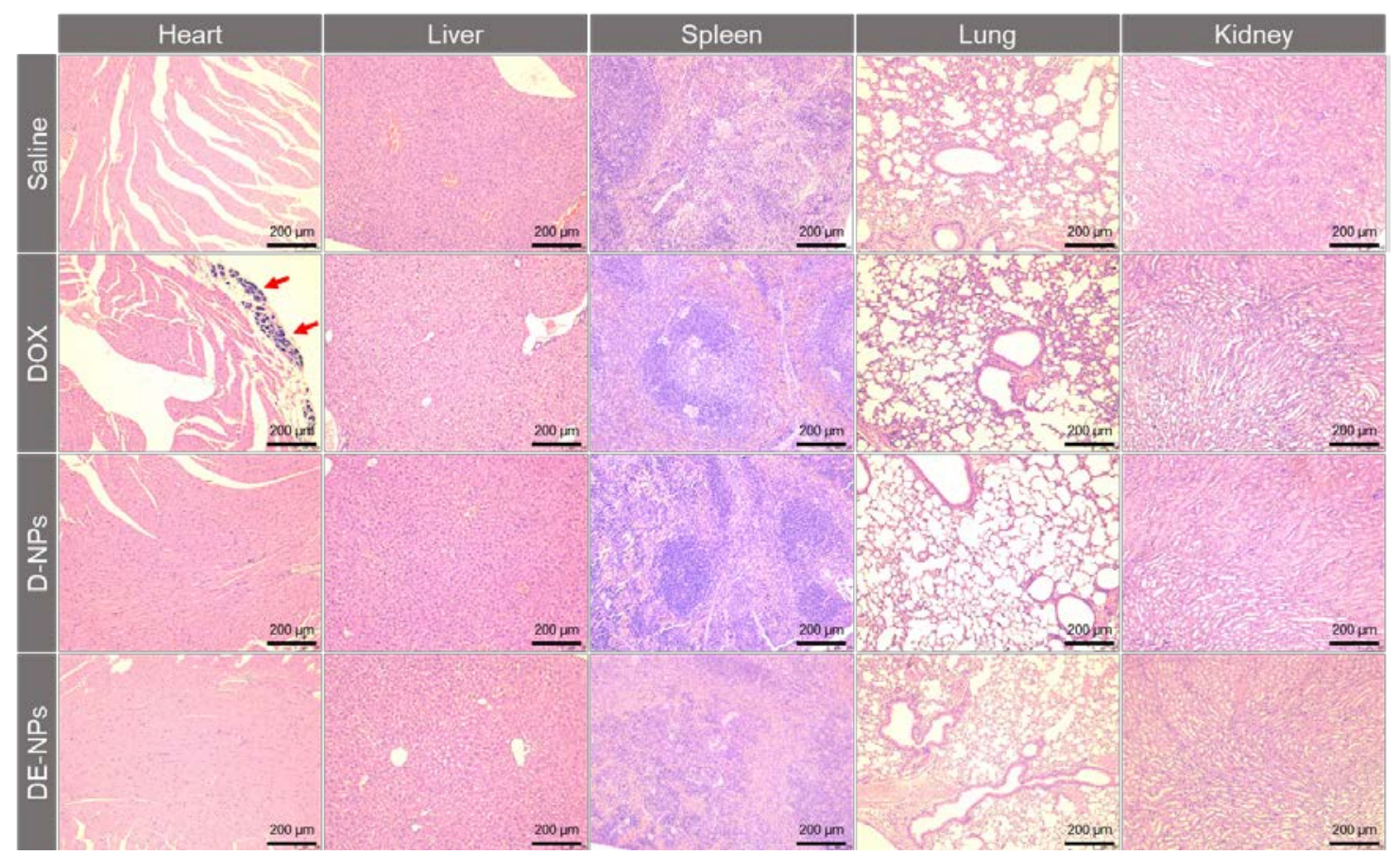

Figure S22. H\&E staining images of different organs from tumor-bearing mice on day 18 . 